\title{
Determination of the systematic position of the genus Asticcacaulis Poindexter by a polyphasic analysis
}

\author{
Wolf-Rainer Abraham, ${ }^{1}$ Carsten Strömpl, ${ }^{1}$ Marc Vancanneyt, ${ }^{2}$ \\ Heinrich Lünsdorf ${ }^{1}$ and Edward R. B. Moore ${ }^{1}$
}

\footnotetext{
1 GBF - German Research Centre for Biotechnology, Dept Microbiology, Mascheroder Weg 1, D-38124 Braunschweig, Germany

2 Laboratorium voor Microbiologie, Universiteit Gent, Gent, Belgium
}

Author for correspondence: Wolf-Rainer Abraham. Tel: +49 5316181 419. Fax: +49 5316181411. e-mail:wab@gbf.de

\begin{abstract}
The genus Asticcacaulis, to date, comprises two species of unicellular, stalked bacteria, developing a stalk at a site which is not coincidental with the centre of the pole of the cell. Multiplication is similar to that demonstrated by the prosthecate species of the genera Caulobacter, Brevundimonas and Maricaulis. A polyphasic approach, comprising 165 rRNA gene sequencing, lipid analysis and $\mathrm{NaCl}$ tolerance characterizations, was used to clarify the taxonomy of the two Asticcacaulis species. From the analysis of the 16S rRNA gene sequences, a close phylogenetic relationship between the species that comprise the genera Asticcacaulis, Caulobacter and Brevundimonas could be deduced wherein the three genera form three distinct branches. The individual genera could also be discerned by different characteristic polar lipids. The species of Asticcacaulis differed from species of Caulobacter and Brevundimonas by the lack of 1,2diacyl-3-0-[6'-phosphatidyl- $\alpha$-D-glucopyranosyl]glycerol. They also did not contain 1,2-di-O-acyl-3-O-[D-glucopyranosyl-(1 $\rightarrow 4)$ - $\alpha$-D-glucuronopyranosyl]glycerol, which is found in most Brevundimonas species but not in strains of the genus Caulobacter. The morphological differences seen between the two species Asticcacaulis excentricus and Asticcacaulis biprosthecium are mirrored by the observed 165 rDNA sequence similarity value of $95 \cdot 3 \%$, which is relatively low compared to the interspecies similarity values observed within the genera Brevundimonas or Caulobacter.
\end{abstract}

Keywords: Asticcacaulis excentricus, Asticcacaulis biprosthecium, Caulobacter, Brevundimonas, polyphasic approach

\section{INTRODUCTION}

The genus Asticcacaulis, to date, comprises two species of unicellular, stalked bacteria, developing a stalk at a site which is not coincidental with the centre of the pole of the cell. The stalk does not possess adhesive material; this is produced instead by the cell at or near the pole of the cell adjacent to the stalk. Multiplication is similar to that demonstrated by species of the genus Caulobacter, i.e. by division of the stalked cell, giving rise to a swarmer cell which is motile by means of a

\footnotetext{
Abbreviations: CID, collision-induced dissociation; FAB-MS, fast atom bombardment-MS; PG, phosphatidylglycerol.

The EMBL accession numbers for the 16S rRNA gene sequences of Asticcacaulis excentricus DSM $4724^{\top}$ and Asticcacaulis biprosthecium DSM $4723^{\top}$ are $A J 247194$ and $A J 247193$, respectively.
}

single flagellum arising at an excentric position on the pole of the cell. This cell subsequently develops a stalk and enters the immotile vegetative phase. All strains have been isolated from freshwater samples.

The genus was named Asticcacaulis (Gr. privative without; Anglo-Saxon sticca stick; L. caulis stalk) because of the non-sticking stalks of the cells of the first described species, Asticcacaulis excentricus (Poindexter, 1964). A second species, Asticcacaulis biprosthecium, was described by Pate et al. (1973). Since then, few reports on strains of this genus have been published and molecular data on the two type strains are scarce. Recently, Sly et al. (1999) reported on the 16S rRNA gene sequences of the two Asticcacaulis type strains. In the course of our study of caulobacteria (Abraham et al., 1997, 1999), an interest in the lipids of the Asticcacaulis spp. and the phylogeny 
of this genus was generated. The $16 \mathrm{~S}$ rDNA sequences, which do not coincide with the sequences reported by Sly et al. (1999), and the polar lipids of A. excentricus and $A$. biprosthecium and their comparison with those of other caulobacteria are reported in this study.

\section{METHODS}

Organisms and culture conditions. A. excentricus DSM $4724^{\mathrm{T}}$ and $A$. biprosthecium DSM $4723^{\mathrm{T}}$ were obtained from the German Collection of Microorganisms and Cell Cultures (DSMZ). The type strains of the type species of the genera Caulobacter (Caulobacter vibrioides DSM 9893' ) and Brevundimonas (Brevundimonas diminuta LMG 2089 $)$ were obtained from the DSMZ and the Laboratorium voor Microbiologie, Universiteit Gent, Belgium, respectively. All strains were grown in PYEM medium $(2 \mathrm{~g}$ peptone, $2 \mathrm{~g}$ yeast extract, $0.5 \mathrm{~g} \mathrm{NH}_{4} \mathrm{Cl}, 11$ deionized water). After autoclaving and cooling, $5 \mathrm{ml}$ riboflavin $\left(0.2 \mathrm{mg} \mathrm{ml}^{-1}\right.$, sterile filtered), $2 \mathrm{ml} \mathrm{50 \%}$ glucose (sterile), $1 \mathrm{ml} 20 \% \mathrm{MgSO}_{4}$ (sterile) and $1 \mathrm{ml} 10 \% \mathrm{CaCl}_{2}$ (sterile) were added. The strains were incubated in 21 flasks at $30^{\circ} \mathrm{C}$, shaken at 100 r.p.m.; the biomass was harvested by centrifugation, at late exponential growth phase, after $72 \mathrm{~h}$.

Electron microscopy. Cells of the late growth phase were negatively stained with uranyl acetate $(2 \%, \mathrm{pH} 4.5)$ for analysis by transmission electron microscopy as described in detail by Yakimov et al. (1998).

16S rDNA sequencing and analysis. Cell pellets from lipid analysis were suspended in $100 \mu \mathrm{l}$ TE buffer $(10 \mathrm{mM}$ Tris/ $\mathrm{HCl}, 1 \mathrm{mM}$ EDTA, $\mathrm{pH} \mathrm{8.0)} \mathrm{and} \mathrm{boiled} \mathrm{for} 5 \mathrm{~min}$. The lysate was centrifuged briefly and $1 \mu \mathrm{l}$ supernatant, containing DNA, was used for PCR; the 16S rRNA genes were targeted using a forward primer, hybridizing at positions 8-27, and a reverse primer, hybridizing at the complement of positions 1525-1541 (Escherichia coli 16S rRNA gene sequence numbering). PCR was carried out using conditions described previously (Karlson et al., 1993). Amplified 16S rDNA was purified using Microcon 100 microconcentrators (Amicon) and the nucleotide sequences were determined using an Applied Biosystems 373A DNA sequencer and the protocols recommended by the manufacturer (Perkin Elmer) for Taq polymerase-initiated cycle sequencing with fluorescent dye-labelled dideoxynucleotides. The sequencing primers have been described previously (Lane, 1991). The nucleotide sequences were aligned with reference 16S rRNA and 16S rRNA gene sequences using evolutionarily conserved primary sequence and secondary structure (Gutell et al., 1985) as references. Evolutionary distances (Jukes \& Cantor, 1969) were calculated from sequence-pair dissimilarities using only homologous, unambiguously determined nucleotide positions (ambiguous nucleotide positions were excluded from sequence pair comparisons). Phylogenetic trees were constructed using the programs of the PHYLIP package (Felsenstein, 1989).

Whole-cell fatty acid analysis. Cells were grown for $48 \mathrm{~h}$ on PYEM agar plates (1.5\% agar). Cells were saponified [15\% (w/v) $\mathrm{NaOH}, 30 \mathrm{~min}, 100^{\circ} \mathrm{C}$ ], methylated to fatty acid methyl esters (FAMEs) (methanolic $\mathrm{HCl}, 10 \mathrm{~min}, 80^{\circ} \mathrm{C}$ ) and extracted [hexane/methyl-tert-butyl ether $(1: 1, \mathrm{v} / \mathrm{v})$ ] as described in detail by Osterhout et al. (1991). FAMEs were analysed by GC. Separation of FAMEs was achieved using fused-silica capillary column $(25 \mathrm{~m}$ by $0.2 \mathrm{~mm})$ with crosslinked $5 \%$ phenyl-methyl silicone (film thickness $0.33 \mu \mathrm{m}$; HP Ultra2). The computer-controlled parameters were the same as those described by Osterhout et al. (1991). The instrument was equipped with an FID and an autosampler. $\mathrm{H}_{2}$ served as the carrier gas.

Polar lipid fatty acid analysis. Lipids were extracted, using a modified Bligh-Dyer procedure (Bligh \& Dyer, 1959) and FAMEs were generated and analysed by GC, as described previously (Abraham et al., 1997).

Fast atom bombardment (FAB)-MS. FAB-MS in the negative mode was performed on the first of two mass spectrometers of a tandem high-resolution instrument of $E_{1} B_{1} E_{2} B_{2}$ configuration (JMS-HX/HX110A; JEOL Tokyo) at $10 \mathrm{kV}$ accelerating voltage. Resolution was set to $1: 1500$. The JEOL FAB gun was operated at $6 \mathrm{kV}$ with xenon as the FAB gas. A mixture of triethanolamine and tetramethylurea $(2: 1$, $\mathrm{v} / \mathrm{v}$ ) was used as matrix. In tandem MS, negative daughter ion spectra were recorded using all four sectors of the tandem mass spectrometer. High energy collision-induced dissociation (CID) took place in the third field-free region. Helium served as the collision gas at a pressure sufficient to reduce the precursor ion signal to $30 \%$ of the original value. The collision cell was operated at ground potential. Resolution of MS2 was set to 1/1000. FAB-CID spectra (linked scans of MS2 at constant B/E ratio) were recorded at $300 \mathrm{~Hz}$ filtering with a JEOL DA 7000 data system.

Salt-tolerance characterization. Type strains were grown in $20 \mathrm{ml}$ PYEM medium amended with $0,5,10,20,30,40$, 60,80 or $100 \mathrm{~g} \mathrm{NaCl}^{-1}$. The OD of the cell suspension (measured at $600 \mathrm{~nm}$ ) was set to $0 \cdot 3$ at the beginning of the experiment and determined at time intervals over $2 \mathrm{~d}$. The differences between these measurements were used to determine salt tolerances.

\section{RESULTS}

\section{S rRNA gene sequences}

The nucleotide sequences of the nearly complete $16 \mathrm{~S}$ rRNA genes have been determined for the type strains of the two species of Asticcacaulis. A primer pair annealing at the $5^{\prime}$ - and $3^{\prime}$-termini of the 16S rRNA genes was used allowing the amplification and sequence determination of 1416 nucleotide positions (approx. 97\% of the complete gene as estimated by comparison with the 16S rRNA gene sequence of $E$. coli). The sequence data have been deposited with the EMBL database (Stoesser et al., 1998) under accession nos AJ247194 (A. excentricus DSM 4724 ${ }^{\mathrm{T}}$ ) and AJ247193 (A. biprosthecium DSM 4723 ${ }^{\mathrm{T}}$ ). The $16 \mathrm{~S}$ rRNA gene sequences of $A$. excentricus and $A$. biprosthecium had $95.3 \%$ sequence similarity and comprised a distinct phylogenetic branch separate from Caulobacter and Brevundimonas species. Fig. 1 presents a dendrogram depicting the estimated phylogenetic/taxonomic positions of Asticcacaulis species, with respect to each other and the described species of the Caulobacter and Brevundimonas genera within the $\alpha$-Proteobacteria.

\section{Gas chromatographic analysis of FAMEs}

The cellular fatty acid compositions of both Asticcacaulis type strains are shown in Table 1. Dominant fatty acids for both species (the relative 


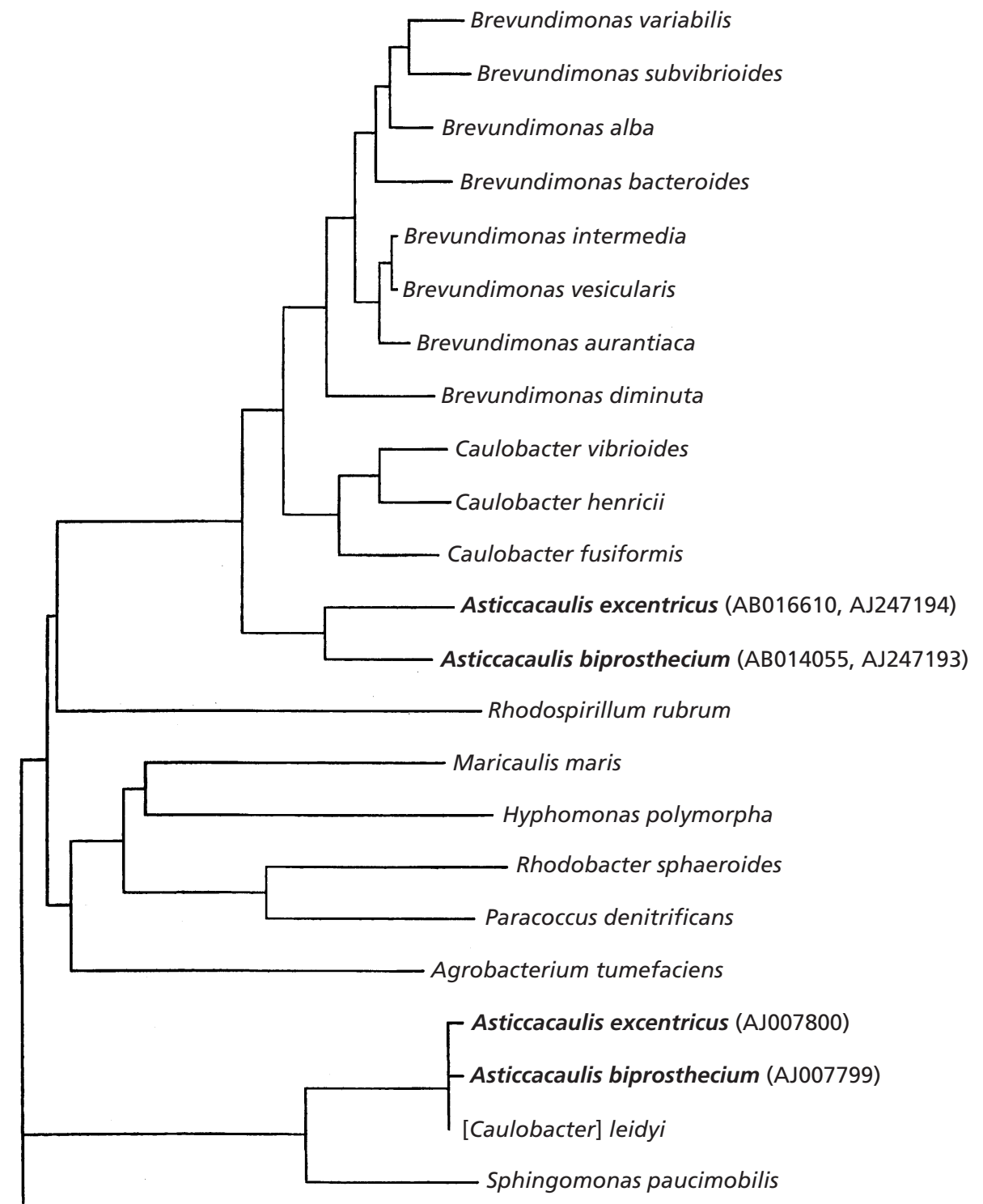

0.01

Rickettsia typhi

Fig. 1. Unrooted dendrogram showing the estimated phylogenetic relationships based on 165 rDNA sequence comparisons of the species of the genera Asticcacaulis, Caulobacter and Brevundimonas within the context of the $\alpha$ Proteobacteria. The dendrogram was generated using the FITCH algorithm in the PHYLIP package. The scale bar represents evolutionary distance as the mean number of fixed mutations per nucleotide position since the point of branching. Rickettsia typhi is included as an outgroup. The sequence data not resulting from this study were obtained from the DDBJ/EMBL/GenBank and/or RDP databases under the following accession numbers: Rickettsia typhi str. Wilmington (L36221), Sphingomonas paucimobilis DSM 1098 ${ }^{\top}$ (X72722), Rhodospirillum rubrum str. ATH 1.1.1 (RDP), Agrobacterium tumefaciens bv.1 NCPPB 2437 (D14500), Rhodobacter sphaeroides IL 106 (D16424), Paracoccus denitrificans LMG 4218 (X69159), Caulobacter vibrioides DSM 9893 ${ }^{\top}$ (AJ227754), Caulobacter henricii ATCC 15253 ${ }^{\top}$ (AJ227758), Caulobacter fusiformis ATCC 15257 ${ }^{\top}$ (AJ227759), Brevundimonas diminuta LMG 2089 ${ }^{\top}$ (AJ227778), Brevundimonas vesicularis LMG $2350^{\top}$ (AJ227780), Brevundimonas bacteroides LMG 15096 ${ }^{\top}$ (AJ227782), Brevundimonas variabilis ATCC 15255 ${ }^{\top}$ (AJ227783), Brevundimonas subvibrioides LMG 14903 ${ }^{\top}$ (AJ227784), Brevundimonas alba DSM 4736 ${ }^{\top}$ (AJ227785), Brevundimonas intermedia ATCC 15262 ${ }^{\top}$ (AJ227786), Brevundimonas aurantiaca DSM 4731 ${ }^{\top}$ (AJ227787), [Caulobacter] leidyi ATCC 15260 ${ }^{\top}$ (AJ227812), Hyphomonas polymorpha DSM 2665 ${ }^{\top}$ (AJ227813), Maricaulis maris ATCC 15268 ${ }^{\top}$ (AJ227802), Asticcacaulis excentricus DSM 4724 ${ }^{\top}$ (AB016610, AJ247194) and ACM 1263 ${ }^{\top}$ (AJ007800), Asticcacaulis biprosthecium DSM 4723 (AB014055, AJ247193) and ACM 2498' (AJ007799).

percentages for $A$. biprosthecium and A. excentricus, respectively, are given in parentheses) are: $12: 13-\mathrm{OH}$ $(7 \cdot 5$ and $4.2 \%) ; 15: 0(2 \cdot 9$ and $2 \cdot 3 \%) ; 16: 0(20 \cdot 8$ and
$17 \cdot 3 \%)$; summed feature $4(13 \cdot 8$ and $7 \cdot 4 \%) ; 17: 1 \omega 6 c$ $(1.0$ and $1.5 \%) ; 18: 1 \omega 7 c(40.7$ and $57.7 \%)$; and $11-$ methyl $18: 1 \omega 7 c(3.3$ and $3.6 \%)$. A. biprosthecium 
Table 1. Percentage whole-cell fatty acid composition in Asticcacaulis species and related taxa

Only the fatty acids counting for more than $1.0 \%$ in one of the strains studied were indicated. Abbreviations: - , not detected; tr, trace amount (less than $1 \cdot 0 \%$ ); ECL, equivalent chain-length (the identity of the fatty acid is not known); SF, summed feature.

\begin{tabular}{|c|c|c|c|c|c|c|c|c|c|c|c|c|c|c|c|c|c|c|c|c|}
\hline Species & $\begin{array}{c}\text { ECL } \\
11 \cdot 798\end{array}$ & $12: 0$ & $\begin{array}{c}12: 0 \\
3-\mathrm{OH}\end{array}$ & $\begin{array}{c}12: 1 \\
3-\mathrm{OH}\end{array}$ & 14:0 & $15: 0$ & $16: 0$ & SF $4^{*}$ & $\begin{array}{c}16: 1 \\
2-\mathrm{OH}\end{array}$ & $\begin{array}{l}16: 0 \\
\text { iso }\end{array}$ & $17: 0$ & $\begin{array}{l}17: 1 \\
\omega 6 c\end{array}$ & $\begin{array}{l}17: 1 \\
\omega 8 c\end{array}$ & $\begin{array}{c}\text { ECL } \\
17 \cdot 897\end{array}$ & SF $7 \dagger$ & $\begin{array}{c}\text { ECL } \\
18 \cdot 080\end{array}$ & $\begin{array}{c}\text { ECL } \\
18 \cdot 140\end{array}$ & $\begin{array}{c}\text { ECL } \\
18 \cdot 797\end{array}$ & 19:0 & $\begin{array}{c}19: 0 \\
\text { cyclo } \omega 8 c\end{array}$ \\
\hline $\begin{array}{l}\text { A. biprosthecium } \\
\text { DSM } 4723^{\mathrm{T}}\end{array}$ & $\operatorname{tr}$ & 2.5 & - & $7 \cdot 5$ & $1 \cdot 1$ & $2 \cdot 9$ & $20 \cdot 8$ & 13.8 & - & - & - & 1.0 & - & - & $40 \cdot 7$ & $3 \cdot 3$ & $3 \cdot 2$ & - & - & - \\
\hline $\begin{array}{l}\text { A. excentricus } \\
\text { DSM } 4724^{\mathrm{T}}\end{array}$ & - & - & - & $4 \cdot 2$ & - & $2 \cdot 3$ & $17 \cdot 3$ & $7 \cdot 4$ & - & 1.8 & 1.8 & 1.5 & $1 \cdot 1$ & - & $57 \cdot 7$ & 3.6 & - & - & - & - \\
\hline $\begin{array}{l}\text { B. diminuta } \\
\text { LMG } 2089^{\mathrm{T}}\end{array}$ & - & - & 1.5 & $\operatorname{tr}$ & $\operatorname{tr}$ & $7 \cdot 6$ & $10 \cdot 1$ & $1 \cdot 0$ & - & - & $8 \cdot 5$ & $6 \cdot 2$ & $10 \cdot 8$ & $1 \cdot 1$ & 38.7 & - & - & $3 \cdot 1$ & - & $6 \cdot 2$ \\
\hline $\begin{array}{l}\text { C. vibrioides } \\
\text { DSM } 9893^{\mathrm{T}}\end{array}$ & $1 \cdot 6$ & - & $\operatorname{tr}$ & $1 \cdot 2$ & 1.4 & 5.8 & $6 \cdot 0$ & $9 \cdot 4$ & $6 \cdot 1$ & - & $8 \cdot 6$ & $3 \cdot 0$ & $2 \cdot 4$ & - & 35.8 & $4 \cdot 3$ & - & $3 \cdot 7$ & 1.5 & - \\
\hline
\end{tabular}

* Summed feature 4 consisted of one or more of the following fatty acids which could not be separated by the Microbial Identification System: 15:0 iso 2-OH, $16: 1 \omega 7 c$ and $16: 1 \omega 7 t$.

$\uparrow$ Summed feature 7 consisted of one or more of the following fatty acids: $18: 1 \omega 7 c, 18: 1 \omega 9 t$ and $18: 1 \omega 12 t$.

DSM $4723^{\mathrm{T}}$ could be differentiated from A. excentricus DSM $4724^{\mathrm{T}}$ by the presence of small amounts of $12: 0$, $14: 0$, ECL 18.140, and the absence of $16: 0$ iso, 17:0 and $17: 1 \omega 8 c$.

\section{Analysis of the polar lipids}

Glycolipids present in all Asticcacaulis spp. were $\alpha$-Dglucopyranosyl- and $\alpha$-D-glucuronopyranosyl-diacylglycerols, which are also common in Caulobacter, Brevundimonas and some other $\alpha$-Proteobacteria. Using CID-MS, the structures of twelve of the predominant glycolipids listed in Table 2 were identified. The ( - )-FAB mass spectra of the phospholipids of both Asticcacaulis species showed clear differences to those of the type species of Caulobacter and Brevundimonas (Fig. 2). Phospholipids of the Asticcacaulis spp. were of the phosphatidylglycerol (PG) type and five phospholipids could be identified.

\section{Salt-tolerance characterization}

A. biprosthecium DSM $4723^{\mathrm{T}}$ and A. excentricus DSM $4724^{\mathrm{T}}$ showed reduced growth without salt, optimal growth with $5 \mathrm{~g} \mathrm{NaCl}^{-1}$ and no growth at salt concentrations of $30 \mathrm{~g}^{-1}$ or more (Fig. 3). Caulobacter vibrioides DSM $9893^{\mathrm{T}}$, the type species of the genus, showed the same reduced growth without $\mathrm{NaCl}$, optimal growth with $5 \mathrm{~g} \mathrm{NaCl} 1^{-1}$ and no growth above $10 \mathrm{~g}^{-1}$. In contrast, the type strain of Brevundimonas, $B$. diminuta LMG $2089^{\mathrm{T}}$, grew without $\mathrm{NaCl}$, tolerated $\mathrm{NaCl}$ concentrations up to $40 \mathrm{~g}^{-1}$ and did not grow above $80 \mathrm{~g} \mathrm{NaCl}^{-1}$.

\section{DISCUSSION}

The 16S rRNA gene sequence similarities of the species of Asticcacaulis ranged from 91.6 to $92.4 \%$ with species of Caulobacter and a $91.9 \%$ sequence similarity was observed between the 16S rRNA genes of $A$. excentricus (the type species of the genus) and $C$. vibrioides (the type species of Caulobacter). Additionally, the 16S rRNA gene sequence similarities of the species of Asticcacaulis ranged from 92.3 to $94.4 \%$ with species of Brevundimonas and 93.1\% sequence similarity was observed between the 16S rRNA genes of $A$. excentricus and $B$. diminuta (the type species of Brevundimonas).

A high overall similarity was observed between the fatty acid content of Asticcacaulis type strains and those of Caulobacter and Brevundimonas reference strains (Table 1; Abraham et al., 1999). All members of the Asticcacaulis-Caulobacter-Brevundimonas phylogenetic branch are characterized by the dominant fatty acids $16: 0$ and $18: 1 \omega 7 c$ (summed feature 7 in Abraham et al., 1999) and the majority of the reference strains of this group of organisms contain significant amounts of the fatty acids 15:0, summed feature 4,

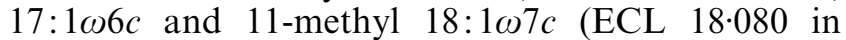
Abraham et al., 1999). Furthermore, a common feature for A. biprosthecium and A. excentricus was the presence of $12: 13-\mathrm{OH}$, a feature that was also observed to be characteristic for Caulobacter species, but not for Brevundimonas taxa. A. biprosthecium could be differentiated from $A$. excentricus by some marked quantitative differences in the dominant fatty acids, by the presence of low amounts of 12:0, 14:0 and ECL 18.140, and by the absence of 16:0 iso, 17:0 and $17: 1 \omega 8 c$ (see above). No 14:0 2-OH was present, a characteristic feature for [Caulobacter] leidyi and members of the genus Sphingomonas (Yabuuchi et al., 1990). The fatty acid data are consistent with the phylogenetic position of the genus Asticcacaulis near to the genera Caulobacter and Brevundimonas and not in the genus Sphingomonas as indicated by Sly et al. (1999).

The species of Asticcacaulis differ from Caulobacter and Brevundimonas by the lack of 1,2-diacyl-3-O- 
Table 2. Polar lipids identified in Asticcacaulis spp. using CID-MS

\begin{tabular}{|cllllll|}
\hline \multicolumn{3}{|c}{ Glycolipid } & & \multicolumn{3}{c|}{ Phospholipid } \\
\cline { 1 - 3 } \cline { 5 - 6 } Mass & Type* $^{*}$ & Fatty acid & & Mass & Type $\dagger$ & Fatty acid \\
& & & & & \\
\hline 730 & MGDOx & $16: 0-15: 0$ & & 734 & PG & $18: 1-15: 0$ \\
742 & MGD & $18: 1-15: 0$ & & 746 & PG & $18: 1-16: 1$ \\
742 & MGDOx & $16: 0-16: 1$ & & 748 & PG & $18: 1-16: 0$ \\
744 & MGD & $17: 0-16: 0$ & & 762 & PG & $18: 1-17: 0$ \\
744 & MGDOx & $16: 0-16: 0$ & & 774 & PG & $18: 1-18: 1$ \\
768 & MGDOx & $18: 1-16: 1$ & & & \\
768 & MGDOx & $18: 2-16: 0$ & & & \\
770 & MGDOx & $18: 1-16: 0$ & & & \\
782 & MGD & $18: 1-18: 1$ & & & \\
782 & MGD & $19: 1-17: 1$ & & & \\
784 & MGD & $19: 1-17: 0$ & & & \\
784 & MGDOx & $18: 1-17: 0$ & & & \\
\hline
\end{tabular}

* MGD, 1,2-di- $O$-acyl-3- $O$ - $\alpha$-D-glucopyranosylglycerol;

MGDOx, 1,2-di- $O$-acyl-3- $O$ - $\alpha$-D-glucuronopyranosylglycerol. $\uparrow \mathrm{PG}$, phosphatidylglycerol.

[6'-phosphatidyl- $\alpha$-D-glucopyranosyl]glycerol (PGL). They also did not contain 1,2-di- $O$-acyl-3- $O$-[D-glucopyranosyl-( $1 \rightarrow 4)-\alpha$-D-glucuronopyranosyl]glycerol (DGL) found in most Brevundimonas strains but not in strains of the genus Caulobacter. Sphingolipids, characteristic for strains of the genus Sphingomonas, were not detected in any of the Asticcacaulis species. There were also differences in the lipid composition within the different lipid types detected. From the lipids found, (17:0-16:0)- and (19:1-17:0)- $\alpha$-D-glucopyranosyl-diacylglycerol, and (16:0-15:0)- and (18:2-16: $0)-\alpha$-D-glucuronopyranosyl-diacylglycerol were not detected in Caulobacter or Brevundimonas strains (70 strains analysed). Phospholipids were PG and, from the five identified lipids, (18:1-17:0)-PG was not found in Caulobacter or Brevundimonas strains (Table 3).

The heterogeneity observed in the 16S rDNA sequences and lipid data of the Asticcacaulis spp. was mirrored in the tolerance to $\mathrm{NaCl}$, resulting in a broad range of tolerances by the individual species to $\mathrm{NaCl}$ concentrations between 10 and $20 \mathrm{~g} \mathrm{l}^{-1}$ (Fig. 3). While A. biprosthecium DSM $4723^{\mathrm{T}}$ showed almost the same $\mathrm{NaCl}$ tolerance as $C$. vibrioides DSM $9893^{\mathrm{T}}, A$. excentricus DSM $4724^{\mathrm{T}}$ tolerated higher $\mathrm{NaCl}$ concentrations. B. diminuta $\mathrm{LMG} 2089^{\mathrm{T}}$ differed from both Asticcacaulis spp. in that it grew well without salt and tolerated much higher $\mathrm{NaCl}$ concentrations than even A. excentricus.

The sequence similarity of $95.3 \%$ between the two described species of Asticcacaulis, which is rather low compared to the similarity values observed between the species of Brevundimonas or the species of Caulobacter (Abraham et al., 1999), suggests that they may be members of separate genera. While bacterial taxonomy cannot be based solely upon 16S rRNA gene sequence data, in many cases sequence comparisons and cluster analysis are able to recognize cases wherein the phylogeny of organisms does not agree with the described taxonomy. However, the level of $16 \mathrm{~S}$ rRNA gene sequence similarity observed between the two species of Asticcacaulis does not allow one to evaluate confidently whether the organisms should be separated in two distinct genera or retained within the same genus. This is supported by the different morphologies and, in part, by the lipid data and the different salt tolerances. However, although the two species, ultimately, may be separated taxonomically, due to the fact that only a single strain for each species has been analysed to date, it is prudent to retain the current classification of the two species within the genus Asticcacaulis until other, more definitive, data provide evidence for reclassification.

\section{Taxonomic implications}

During the course of this study, sequence data for the 16S rRNA genes of the two Asticcacaulis species were submitted, by two separate groups (Sly et al., 1999; Hamada et al., 1998) to the DDBJ/EMBL/GenBank databases. The sequence data determined in the current study were compared with these sequences. The 16S rRNA gene sequence determined in this study for A. excentricus (DSM $4724^{\mathrm{T}} \leftarrow$ ATCC $15261^{\mathrm{T}}$ ) was observed to be identical for all 1416 homologous nucleotide positions for the sequence determined by Hamada et al. (1998) (strain ATCC 15261 ${ }^{\mathrm{T}}$, accession no. AB016610), yet only $85.1 \%$ similar to the sequence determined by Sly et al. (1999) (strain ACM $1263^{\mathrm{T}}=$ ATCC $15261^{\mathrm{T}}$, accession no. AJ007800). The $16 \mathrm{~S}$ rRNA gene sequence determined in this study for $A$. biprosthecium (DSM $4723^{\mathrm{T}} \leftarrow$ ATCC $27554^{\mathrm{T}}$ ) was observed to be identical over all 1406 homologous nucleotide positions for the sequence determined by Hamada et al. (1998) (strain ATCC 27554 ${ }^{\mathrm{T}}$, accession no. AB014055), yet only $84 \cdot 6 \%$ similar to the sequence determined by Sly et al. (1999) (strain ACM $2498^{\mathrm{T}}=$ ATCC 27554 ${ }^{\mathrm{T}}$, accession no. AJ007799). Cluster analysis of the sequences of the strains of this study (as well the sequences determined by Hamada et al., 1998) indicates a phylogenetic relationship of Asticcacaulis species to Caulobacter and Brevundimonas species (Fig. $1)$. This was corroborated by fatty acid data, which were closely related to those of the species of the genera Caulobacter and Brevundimonas while the 2-hydroxy fatty acids, characteristic for the Sphingomonas paucimobilis branch, were absent. However, the sequences determined by Sly et al. (1999) cluster with the sequences of species of Sphingomonas and [Caulobacter] leidyi. These observations suggest that the strains which have been sequenced have been misidentified in at least one case. In the course of this study, the strains were obtained from the DSMZ, cultivated and examined by microscopy to confirm the development of the characteristic prosthecate appendages described for the two species of Asticcacaulis 
(a)

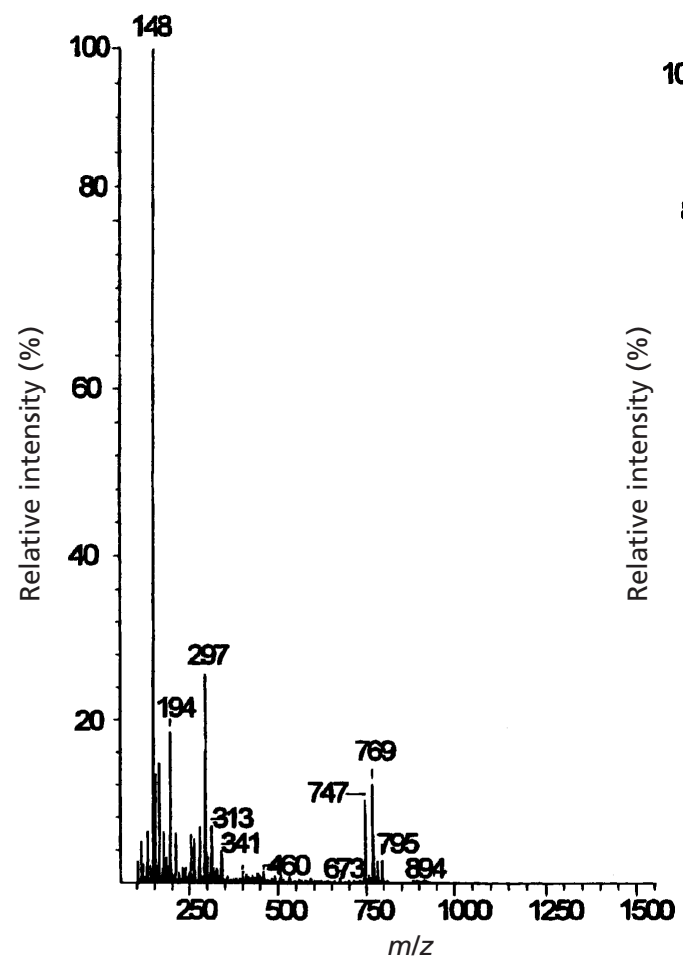

(b)

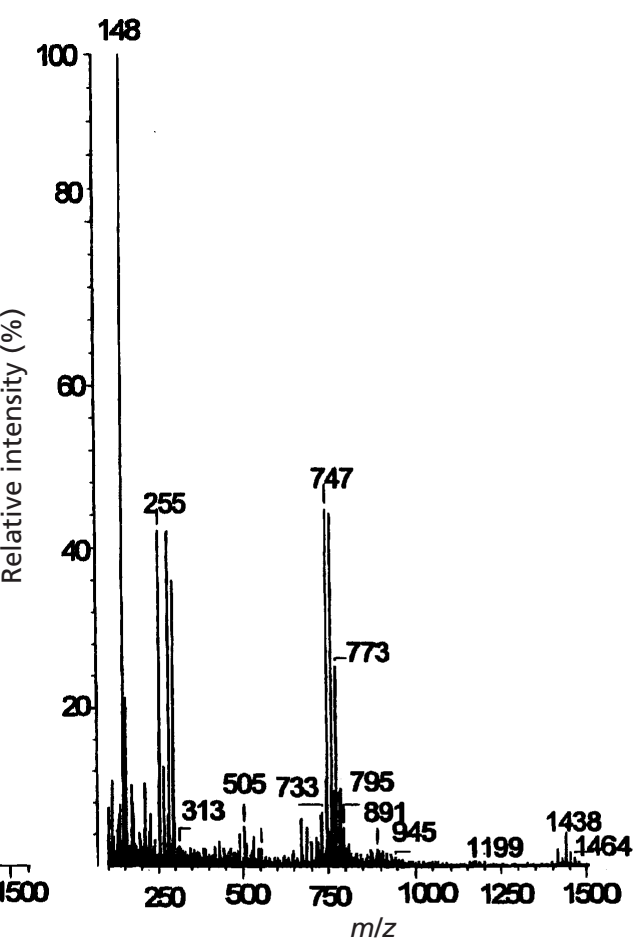

(d)

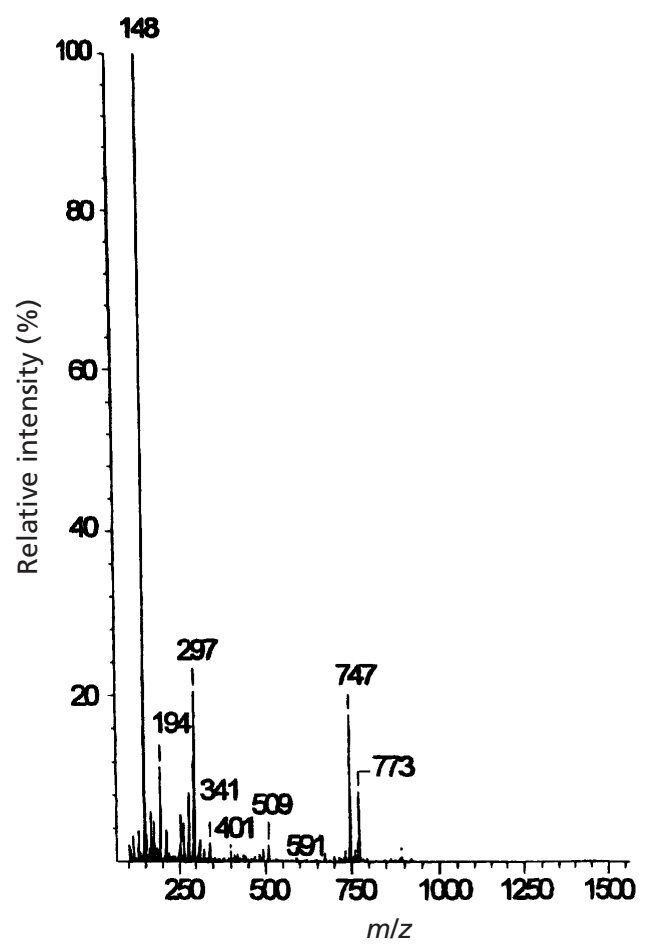

Fig. 2. (-)-FAB mass spectrum of the phospholipid fraction of $A$. biprosthecium DSM $4723^{\top}$ (a), Brevundimonas diminuta LMG $2089^{\top}$ (b), Caulobacter vibrioides DSM $9893^{\top}$ (c) and Asticcacaulis excentricus DSM 4724 (d). In the range $\mathrm{m} / \mathrm{z}$ 250-300 the acyl ions, around $\mathrm{m} / \mathrm{z} 750$ phosphatidylglycerol (PG) ions, between $\mathrm{m} / \mathrm{z}$ 930-990 1,2-di-O-acyl-3-O-[Dglucopyranosyl-(1 $\rightarrow 4)-\alpha$-D-glucuronopyranosyl]glycerol (DGL) and finally around $\mathrm{m} / \mathrm{z}$ 1410-1465 1,2-diacyl-3-O-[6'phosphatidyl- $\alpha$-D-glucopyranosyl]glycerol (PGL) are seen. Note that PGL is only seen in the Caulobacter and Brevundimonas strains, but is absent in the Asticcacaulis strains, and that DGL is only present in the Brevundimonas strain. 


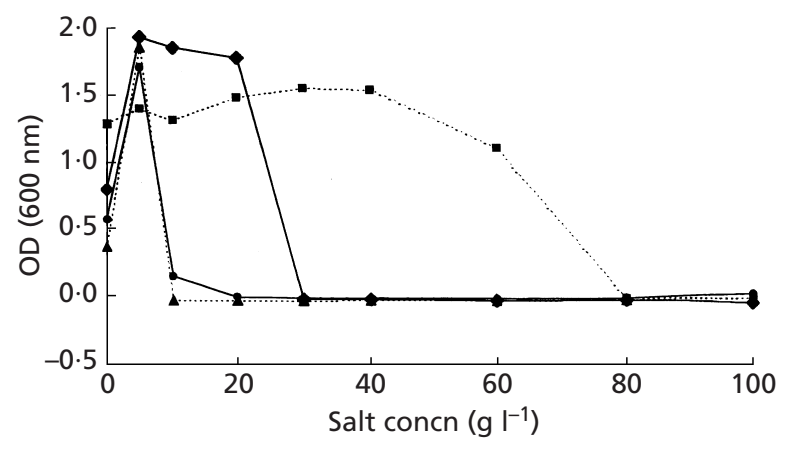

Fig. 3. Comparison of the growth of Asticcacaulis biprosthecium DSM $4723^{\top} \boldsymbol{\Delta}$ and $A$. excentricus DSM $4724^{\top}$ and the type strains of the species Caulobacter (C. vibrioides DSM $9893^{\top}$; 1 ) and Brevundimonas (B. diminuta LMG 2089 ; D) in the presence of different concentrations of $\mathrm{NaCl}$.

(Fig. 4). The cell morphologies of the two strains of this study conformed with the formal descriptions of the respective species. From our examinations, it is concluded that the strains received from DSMZ match, in all aspects, the descriptions given by Poindexter (1964) and Pate et al. (1973). It would be very helpful if ACM and DSMZ would check the authenticity of their Asticcacaulis type strains in order to find out the origin of the differences in the 16S rRNA gene sequences reported by Sly et al. (1999) and in this study.

From the data presented here, a close relationship between the species that comprise the genera Asticcacaulis, Caulobacter and Brevundimonas can be deduced, as was proposed more than three decades ago (Poindexter, 1964). The three genera form three distinct branches within the phylogenetic tree derived from the comparison of the 16S rRNA gene sequences. The individual genera can also be discerned by their characteristic polar lipids. It is noteworthy that the phylogenetic branch including the genera Caulobacter, (a)

(b)

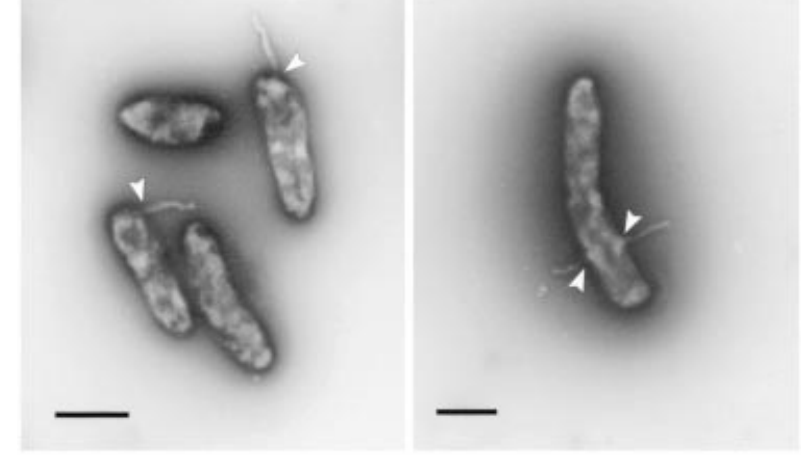

Fig. 4. Morphology of negatively stained late-log cells from Asticcacaulis excentricus DSM $4724^{\top}$ (a) and Asticcacaulis biprosthecium DSM $4723^{\top}$ (b). Arrowheads indicate the offshoots of the prostheca relative to the cell body. Bars, $1 \cdot 1 \mu \mathrm{m}$.

Brevundimonas and Asticcacaulis is comprised predominantly, albeit not exclusively, of organisms capable of developing cellular prosthecate 'holdfast' appendages, although this morphological characteristic is now recognized to occur within diverse phylogenetic lineages. Further, within the phylogenetic branch including the genera Caulobacter, Brevundimonas and Asticcacaulis, species capable of developing prosthecae are recognized to cluster with organisms (i.e. Brevundimonas species, Caulobacter segnis) which have not been observed to produce them (Abraham et al., 1999). Thus, it is clear, from observations of the phylogenetic relationships of 'appendaged bacteria', as derived from 16S rRNA gene sequence comparisons, that the expression of the prosthecateforming phenotype in bacteria is widespread and does not, in itself, comprise a taxonomic signature. However, a specific type of appendage, e.g. the characteristic, subpolar, pseudostalk of Asticcacaulis species, may be confined to species of defined phylogenetic lineages. The genus Asticcacaulis clearly deserves more

Table 3. Polar lipids as biomarkers in Asticcacaulis spp., Brevundimonas spp. and Caulobacter spp. (Abraham et al., 1999)

+++ , Present in all strains; ++ , present in most strains $(>80 \%)$; blank, absent. PG,

Phosphatidylglycerol; SQDG, 1,2-diacyl-3- $O$-sulfoquinovosylglycerol; DGL, 1,2-di- $O$-acyl-

3-O-[D-glucopyranosyl-(1 $\rightarrow 4)$ - $\alpha$-D-glucuronopyranosyl]glycerol; PGL, 1,2-diacyl-3- $O$-[6'-

phosphatidyl- $\alpha$-D-glucopyranosyl]glycerol; MGD, 1,2-di- $O$-acyl-3- $O$ - $\alpha$-D-glucopyranosylglycerol;

MGDOx, 1,2-di- $O$-acyl-3- $O$ - $\alpha$-D-glucuronopyranosylglycerol.

\begin{tabular}{|llccccc|}
\hline Genus & PG & SQDG & DGL & PGL & MGD & MGDOx \\
\hline Asticcacaulis & +++ & & & & +++ & +++ \\
Caulobacter & +++ & & & +++ & +++ & +++ \\
Brevundimonas & +++ & ++ & ++ & +++ & +++ & +++ \\
\hline
\end{tabular}


attention from microbiologists which, hopefully, will result in the characterization of more isolates and a deeper insight into the intra- and intergeneric taxonomy of this interesting group of bacteria as well as its ecological role in the environment.

\section{ACKNOWLEDGEMENTS}

We thank Dagmar Duttmann, Tanja Jeschke, Annette Krüger and Peter Wolff for their excellent technical assistance in culturing the strains, nucleic acid sequencing work and chemical analysis. Ruprecht Christ is thanked for his skilful work at the tandem mass spectrometer. This work was supported by a grant from the German Federal Ministry for Science, Education and Research (Project No. 0319433C).

\section{REFERENCES}

Abraham, W.-R., Meyer, H., Lindholst, S., Vancanneyt, M. \& Smit, J. (1997). Phospho- and sulfolipids as biomarkers of Caulobacter, Brevundimonas and Hyphomonas. Syst Appl Microbiol 20, 522-539.

Abraham, W.-R., Strömpl, C., Meyer, H. \& 8 other authors (1999). Phylogeny and polyphasic taxonomy of Caulobacter species. Proposal of Maricaulis gen. nov. with M. maris (Poindexter) comb. nov. as the type species, and emended description of the genera Brevundimonas and Caulobacter. Int J Syst Bacteriol 49, 1053-1073.

Bligh, E. G. \& Dyer, W. J. (1959). A rapid method for total lipid extraction and purification. Can J Biochem Physiol 37, 911-917.

Felsenstein, J. (1989). PHYLIP - phylogeny inference package (version 3.2). Cladistics 5, 164-166.

Gutell, R. R., Weiser, B., Woese, C. R. \& Noller, H. F. (1985). Comparative anatomy of $16 \mathrm{~S}$-like ribosomal RNA. Prog Nucleic Acid Res Mol Biol 32, 155-216.

Hamada, T., Suzuki, M. \& Harayama, S. (1998). Asticcacaulis excentricus 16S rRNA gene, partial sequence. DDBJ/EMBL/ GenBank databases, accession no. AB016610.
Jukes, T. H. \& Cantor, C. R. (1969). Evolution of protein molecules. In Mammalian Protein Metabolism, vol. 3, pp. 21-132. Edited by H. N. Munro. New York: Academic Press.

Karlson, U., Dwyer, D. F., Hooper, S. W., Moore, E. R. B., Timmis, K. N. \& Eltis, L. D. (1993). Two independently regulated cytochromes P-450 in a Rhodococcus rhodochrous strain that degrades 2-ethoxyphenol and 4-methoxybenzoate. J Bacteriol 175, 1467-1474.

Lane, D. J. (1991). 16S/23S rRNA sequencing. In Nucleic Acid Techniques in Bacterial Systematics, pp. 115-175. Edited by E. Stackebrandt \& M. Goodfellow. Chichester: John Wiley.

Osterhout, G. J., Shull, V. H. \& Dick, J. D. (1991). Identification of clinical isolates of Gram-negative nonfermentative bacteria by an automated cellular fatty acid identification system. $J$ Clin Microbiol 29, 1822-1830.

Pate, J. L., Porter, J. S. \& Jordan, T. L. (1973). Asticcacaulis biprosthecium sp. nov. life cycle, morphology and cultural characteristics. Antonie Leeuwenhoek J Microbiol Serol 39, 569-583.

Poindexter, J. S. (1964). Biological properties and classification of the Caulobacter group. Bacteriol Rev 28, 231-295.

Sly, L. I., Cox, T. L. \& Beckenham, T. B. (1999). The phylogenetic relationships of Caulobacter, Asticcacaulis, and Brevundimonas species and their taxonomic implications. Int $J$ Syst Bacteriol 49, 483-488.

Stoesser, G., Moseley, M. A., Sleep, J., McGowran, M., GarciaPastor, M. \& Sterk, P. (1998). The EMBL nucleotide sequence database. Nucleic Acids Res 26, 8-15.

Yabuuchi, E., Yano, I., Oyaizu, H., Hashimoto, Y., Ezaki, T. \& Yamamoto, H. (1990). Proposals of Sphingomonas paucimobilis gen. nov. and comb. nov., Sphingomonas parapaucimobilis sp. nov., Sphingomonas yanoikuyae sp. nov., Sphingomonas adhaesiva sp. nov., Sphingomonas capsulata comb. nov., and two genospecies of the genus Sphingomonas. Microbiol Immunol 34, 99-119.

Yakimov, M. M., Golyshin, P. N., Lang, S., Moore, E. R. W., Abraham, W.-R., Lünsdorf, H. \& Timmis, K. N. (1998). Alcanivorax borkumii gen. nov., sp. nov., a new hydrocarbon-degrading and surfactant-producing marine bacterium. Int J Syst Bacteriol 48, 339-348. 